\title{
Revisão sistemática da aplicabilidade da entrevista motivacional no tratamento dos transtornos da alimentação
}

\author{
Lais de Mattos Garcia* \\ Ilana Andretta**
}

\begin{abstract}
Resumo
Este estudo tem como objetivo examinar a aplicabilidade e efetividade da Entrevista Motivacional (EM) e suas adaptações no tratamento de transtornos da alimentação (TA). As buscas foram realizadas nas bases de dados eletrônicas Web of Science, PubMed, Lilacs, Cobcrane e Scielo. Foram excluídos artigos que não utilizam a EM como forma única ou associada de intervenção, que avaliam a aplicabilidade da EM em transtornos não relacionados à alimentação e que tratam exclusivamente da aplicabilidade da EM na obesidade. Estudos teóricos, revisões de literatura e produções não disponíveis em texto completo também foram critérios de exclusão. De um total de 85 artigos encontrados quando utilizados os descritores motivational interviewing AND eating disorders, nove artigos foram utilizados. Os resultados observados indicam aumento da motivação, da autoeficácia, da prontidão para mudança e maior adesão ao tratamento de pessoas diagnosticadas com algum transtorno da alimentação quando submetidas ao tratamento com EM e suas adaptações.

Palavras-chave: Entrevista Motivacional. Transtornos da alimentação. Intervenções Motivacionais.
\end{abstract}

\section{Systematic review of Motivational Interviewing applicability in the treatment of eating disorders}

\begin{abstract}
This study aims to examine the applicability and effectiveness of Motivational Interviewing (MI) and its adaptations in the treatment of eating disorders (ED). The scientific research were conducted in electronic databases Web of Science, PubMed, Lilacs, Cobcrane and Scielo. They were excluded articles that do not use MI as single or associated intervention, assessing the applicability of MI in disorders not related to food and dealing exclusively with the applicability of MI in obesity. Theoretical studies, literature reviews and incomplete text productions were also an exclusion criteria. A total of 85 items were found about motivational interviewing AND eating disorders descriptors, then, nine articles were embraced for inclusion. The observed results indicate increased motivation, self-efficacy, readiness for change and greater adherence to the treatment of people diagnosed with any eating disorder when subjected to treatment with MI and their adaptations.

Keywords: Motivational Interviewing. Eating disorders. Motivational interventions.
\end{abstract}

* Graduada em Psicologia pela Universidade do Vale do Rio dos Sinos - UNISINOS (2016/2). Possui experiência nas áreas de prevenção e enfrentamento à violência contra a mulher, dependência química, psicoterapia individual e coordenação de grupos interdisciplinares.

** Possui graduação em Psicologia pela PUCRS (2002), especialista em Psicoterapia cognitivo-comportamental (2011), mestrado em Psicologia Clínica pela PUCRS (2005) e doutorado em Psicologia pela PUCRS (2009), com treinamento avançado em Terapia Cognitiva pelo Beck Institute e em Entrevista Motivacional com William Miller e Theresa Moyers. Atualmente é professora do Programa de Pós-graduação em Psicologia da UNISINOS, coordenadora do grupo de pesquisa Intervenções Cognitivas e Comportamentais: estudo e pesquisa (ICCEP). Endereço de e-mail: ilana.andretta@gmail.com 


\section{Introdução}

Os transtornos da alimentação (TA) têm uma etiologia multifatorial, ou seja, são determinados por uma diversidade de fatores - tais como predisposições genéticas, socioculturais e vulnerabilidades biológicas e psicológicas - que interagem entre si de modo complexo, para produzir e, diversas vezes, perpetuar a doença. (Morgan, Vecchiatti \& Negrão, 2002). Por transtornos da alimentação entendemos uma gama de comportamentos ou pensamentos recorrentes e persistentes em relação à alimentação ou ao ato de comer que, quando na sua execução, causam prejuízo ou sofrimento à pessoa. (Finger \& Guedes, 2016). Um desafio para profissionais da saúde que atendem a pessoas diagnosticadas com transtornos da alimentação é lidar com a ambivalência para mudar, já que estes pacientes, geralmente, não se identificam como tendo um problema. (Blake, Turnbull \& Treasure, 1997).

A Entrevista Motivacional (EM) é um modelo de intervenção clínica que tem como foco a resolução da ambivalência para, assim, aumentar a motivação e eliciar a mudança de comportamentos depreciativos e autodestrutivos. (Miller \& Rollnick, 2001). Para isso, a EM utiliza um conjunto de métodos, empregados para ajudar os indivíduos a aumentar a motivação intrínseca ao explorar e resolver a ambivalência sobre a mudança de um comportamento específico. (Andretta, Meyer, Kuhn \& Rigon, 2014). Este modelo objetiva a motivação do paciente "venha de dentro para fora", visto que se espera que seja ele quem apresente os argumentos para desejar (ou não) mudar determinado comportamento, os quais serão trabalhados em conjunto, de modo que faça sentido para o paciente cada estratégia que venha a ser utilizada. (Andretta, Oliveira, Zambom \& Dornelles 2012).

O terapeuta que utiliza a EM como forma de intervenção deve, essencialmente, buscar fazer sempre perguntas abertas, que não possam ser respondidas com "sim" ou "não", para que o paciente, ao falar sobre seu problema, possa também "se escutar", promovendo a discrepância; escutar reflexivamente, resumindo brevemente o que ele entendeu do que o paciente disse através de uma afirmação que não seja simplesmente uma reprodução do que o paciente falou, mas buscando fazer uma espécie de entendimento acerca daquilo que o paciente traz; encorajar e apoiar o paciente durante o processo terapêutico por meio de elogios e afirmações de apreciação e compreensão; resumir o que foi dito para que o paciente possa melhor acompanhar o processo terapêutico; e eliciar afirmações automotivacionais, por meio de perguntas que evoquem o reconhecimento do problema, as preocupações do paciente e sua intenção de mudar. (Miller \& Rollnick, 2001). Desta forma, entende-se que a EM pode ser capaz de auxiliar nos processos de mudanças comportamentais, trabalhando, assim, a resolução da ambivalência. (Andretta, Oliveira, Zambom \& Dornelles 2012).

Para o bom entendimento dos objetivos desta revisão, que serão elencados a seguir, faz-se necessário introduzir o conceito do Modelo Transteórico de Mudança de Comportamento (MTT), proposto por Prochaska e DiClemente na década de 80. (Prochaska \& DiClemente, 1982). Conforme Prochaska, DiClemente e Norcross (1992), o MTT busca uma melhor compreensão da motivação e se baseia na avaliação de estágios motivacionais pelos quais o sujeito transita. Estes estágios de motivação para a mudança permitem que entendamos quando mudanças particulares, intenções e comportamentos podem ocorrer. (Szupzinski \& Oliveira, 2008). São eles:

1. Pré-contemplação, quando o indivíduo não considera a possibilidade de mudança do seu comportamento, pois não o vê como sendo um problema;

2. Contemplação, marcado pela ambivalência entre continuar com seu comportamento atual ou modificá-lo - o paciente já enxergou o problema, mas ainda está na dúvida quanto a mudar seu comportamento;

3. Preparação, quando o paciente decide pela mudança e percebe que este comportamento o traz prejuízos, então ele começa a elaborar estratégias para mudar efetivamente este comportamento;

4. Ação, estágio no qual o paciente começa a colocar em prática as estratégias que elaborou no estágio anterior; e,

5. Manutenção, quando objetivo é a prevenção à recaída (retorno ao comportamento "problema"). (Prochaska, DiClemente \& Norcross, 1992).

Apesar de similares conceitualmente falando, é importante não confundir a EM com o MTT, ou mesmo inferir que um tenha sido pensado a partir do outro. Enquanto o MT'T se destina a fornecer um modelo conceitual abrangente de como e porque as mudanças ocorrem, a EM é um método clínico específico para aumentar a motivação pessoal para mudar. (Miller \& Rollnick, 2009).

A combinação da EM com a teoria do MTT dá origem à Motivational Enhancement Therapy (MET), também conhecida como Entrevista Motivacional Breve, que foi pensada para ser uma intervenção breve em intensidade e 
duração. (Sales \& Figlie, 2009). A MET tem como objetivo determinar em qual estágio de motivação o paciente se encontra para ajudar, utilizando a EM, no movimento deste através de tais estágios, alcançando, desta forma, o objetivo final da mudança desejada. (Feld, Woodside, Kaplan, Olmsed \& Carter, 2001). Assim como a MET, a Terapia de Motivação e Prontidão (Readiness and Motivation Therapy - RMT) também se caracteriza por ser uma intervenção motivacional que surge como adaptação da EM. Ela identifica o estágio de mudança no qual o indivíduo se encontra e, então, intervém utilizando os princípios da EM. (Geller, Brown \& Srikameswaran, 2011).

Nesta revisão, será examinada a efetividade das intervenções que incluem a utilização da EM e sua adaptação na MET e RMT no tratamento dos transtornos da alimentação. Para isso, buscar-se-á contemplar os seguintes objetivos: (1) examinar o contexto e a efetividade da utilização da EM e suas adaptações no tratamento de pessoas com transtornos da alimentação; e (2) identificar possíveis limitações e/ou obstáculos neste processo.

\section{Metodologia}

O presente estudo trata-se de uma revisão sistemática da literatura acerca da aplicabilidade da Entrevista Motivacional no tratamento dos transtornos da alimentação. Conforme Linde e Willich (2003), revisões sistemáticas são projetadas para evitar preconceitos e trazer os resultados e conclusões de maneira objetiva. Esta revisão basear-se-á no Protocolo PRISMA, que consiste em um checklist com 27 itens e um fluxograma de quatro etapas e tem como objetivo auxiliar na construção de revisões sistemáticas e meta-análises. (Moher, Liberati, Tetzlaff \& Altman, 2009).

Foi realizada uma busca de referências bibliográficas nas bases de dados Web of Science, PubMed, Lilacs, Cohcrane e Scielo. Como descritores para a busca, foram utilizados os seguintes termos: motivational interviewing e eating disorders, tendo-se utilizado como ferramenta de truncagem a palavra $A N D$.

Foram selecionados os estudos que se propuseram a avaliar a aplicabilidade da Entrevista Motivacional no tratamento de diferentes transtornos da alimentação (transtorno de compulsão alimentar - TCAP, anorexia nervosa - $A N$, bulimia nervosa - $B N$, e transtorno alimentar não especificado - EDNOS). Os transtornos alimentares Pica, Transtorno de Ruminação, Transtorno Alimentar Restritivo/Evitativo e outro transtorno alimentar especificado não foram observados nos estudos incluídos.

Foram excluídos artigos que não utilizam a EM como forma única ou associada de intervenção, assim como foram excluídos artigos que avaliam a aplicabilidade da EM em transtornos não relacionados à alimentação, como dependência química e ansiedade, por exemplo. Foram também excluídos artigos que tratam exclusivamente da aplicabilidade da EM na obesidade, visto que, conforme o DSM-5 (American Psychiatry Association, 2014), esta condição não se enquadra como um transtorno mental, pois existe uma gama de fatores genéticos, fisiológicos, comportamentais e ambientais que variam entre os indivíduos e contribuem para o desenvolvimento da obesidade, os quais excluem ela deste enquadre.

Os critérios de seleção incluíram artigos empíricos que foram publicados em periódicos científicos nos quais foi verificada a aplicabilidade e eficácia da EM e suas adaptações na MET e na RMT no tratamento dos transtornos da alimentação. Estudos teóricos, revisões de literatura e produções cujo texto completo não estava disponível online não foram incluídos para esta revisão.

\section{Resultados}

$\mathrm{Na}$ base de dados Web of Science, foram encontrados um total de 70 artigos; utilizando-se os mesmos descritores, foram encontrados 34 artigos na PubMed, um artigo na Lilacs, um artigo na Cohcrane e, na Scielo a busca não resultou em nenhum artigo, totalizando 106 artigos. Os critérios de exclusão e o número de artigos incluídos e excluídos podem ser observados no seguinte fluxograma.

Ao final desta busca, que resultou em 85 artigos - descartando, neste número, os artigos duplicados nas diferentes bases de dados pesquisadas -, foram finalmente selecionados nove artigos científicos que avaliaram empiricamente a aplicabilidade da EM e suas adaptações na MET e RMT no tratamento dos transtornos da alimentação. Após a revisão, os dados pertinentes foram extraídos e agrupados na tabela abaixo. A amostra (participantes) de cada estudo, assim como a metodologia utilizada e a duração da intervenção também se encontram explicitados na tabela na página seguinte.

\section{4 discussão dos resultados}

Nesta revisão foi examinada a aplicabilidade e efetividade da Entrevista Motivacional (EM) e suas adaptações na Entrevista Motivacional Breve (Motivational Enhancement Therapy - MET), na Terapia de Motivação e Prontidão (Readiness and Motivation Therapy - RMT) e no tratamento dos transtornos da alimentação (TA). Tais intervenções motivacionais foram utilizadas de forma individual ou grupal e não foram elencadas diferenças nos resultados entre os diferentes transtornos da alimentação 
FIGURA 1 - Fluxograma dos artigos revisados

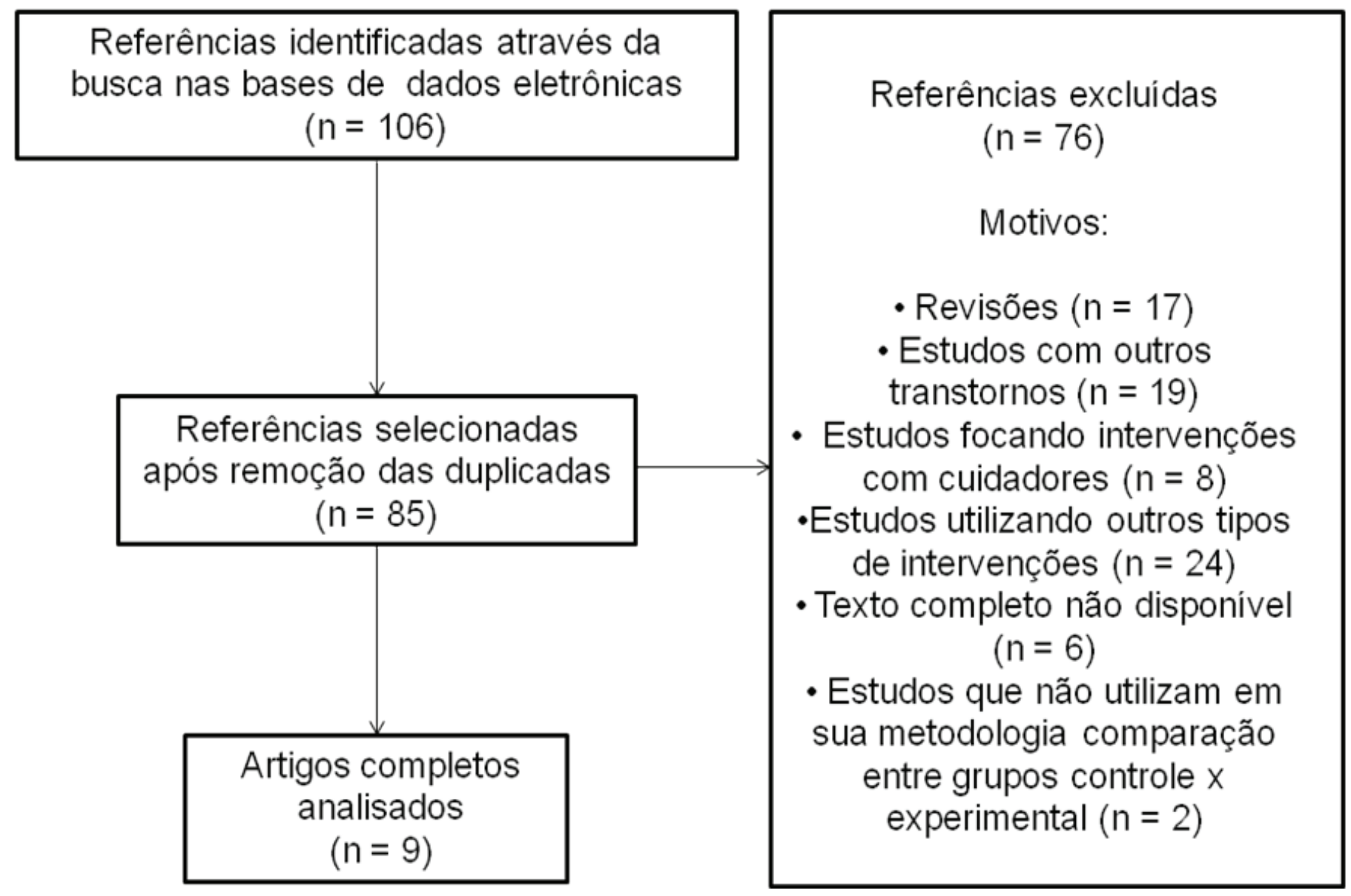

que foram observados nos estudos revisados.

Dos nove estudos revisados, foi possível observar que apenas no estudo de Katzman, Bara-Carril, Rabe-Hesketh, Schmidt, Troop e Treasure (2010) não houve diferenças significativas entre o grupo que recebeu intervenções motivacionais e o que não as recebeu; em todos os grupos foi possível observar benefícios em relação à prontidão para mudança e adesão ao tratamento. Neste estudo, diferentemente dos outros oito, os três grupos que foram comparados receberam algum tipo de intervenção para além de apenas um manual de autoajuda ou o tratamento usualmente realizado no local, visto que a comparação foi realizada entre um grupo que recebeu a MET individual, outro que recebeu a MET em grupo, e um terceiro, que recebeu a Terapia Cognitivo-Comportamental (TCC) em grupo.

O estudo de Vella-Zarb, Mills, Westra, Carter e Keating (2015) comparou os resultados entre um grupo no qual foi utilizada a EM de forma individual com outro grupo, que utilizou uma intervenção psicoeducativa. Em ambos os grupos deste estudo foi disponibilizado, também, um manual de autoajuda, o qual contempla in- formações sobre o Transtorno de Compulsão Alimentar Periódica. Foi possível verificar que os participantes do grupo que utilizavam a EM como forma de intervenção mostraram um aumento significativo na prontidão para mudança e no aumento da autoeficácia quando comparados ao início do tratamento. Quanto aos participantes do grupo que recebeu somente psicoeducação não houve engajamento e nem demonstração de vontade de mudar.

Foi possível identificar nos estudos de Hötzel, Von Brachel, Schmidt, Rieger, Kosfeld, Hechler, Schulted e Vocks (2014), de Weiss, Mills, Westra e Carter (2013), de Wade, Frayne, Edwards, Robertson e Gilchrist (2009), e no de Cassin, Von Ronson, Heng, Brear e Wostowicz (2008), que utilizaram a EM de forma individual no grupo experimental e não a utilizaram no controle, que a autoeficácia, a prontidão para a mudança e a adesão ao tratamento foram mais fortes nos participantes do grupo que recebeu a intervenção motivacional.

Nos três estudos (Geller, Brown \& Srikameswakan, 2011; Dean, Touyz, Rieger \& Thornson, 2008; e Dunn, Neighbors \& Larimer, 2006) que se propuseram a avaliar a efetividade da MET e da RMT de forma individual ou 


\section{Tabela 1 - Artigos Revisados}

Estudo

Amostra
Vella-Zarb, R. et al. (2015)

45 participantes maiores de 18 anos diagnosticados com TCAP.
Hötzel, K. et Weiss, C. et al. al. (2014) (2013)
32 pacientes diagnosticados com algum transtorno da alimentação .

\section{Grupo controle/ experimental \\ Grupo 1: 60' de EM uma vez por semana \\ seguidos por um manual de autoajuda. \\ Grupo 2: 60' de psi coeducação seguidos por um manual de autoajuda.}

Grupo 1: 45' de intervenção motivacional online por meio do programa ESS-KIMO uma vez por semana.

Grupo 2: lista de espera para receber a intervenção online após a conclusão do grupo 1 .
Duração

Principais resultados
A EM aumentou significativamente a prontidão para a mudança e a autoeficácia em relação à capacidade de controlar a compulsão alimentar, ao passo que a psicoeducação não o fez.
Foi observado maior aumento na motivação do grupo 1 em relação ao grupo 2, além de uma redução mais evidente dos sintomas dos transtornos da alimentação no grupo 1 em uma comparação do pré ao póstratamento.
Grupo 1: 16 participantes aleatoriamente designados para quatro sessões individuais semanais de EM com 50' de duração cada.

Grupo 2: 16 participantes foram atribuídos ao tratamento de costume e permaneceram em uma lista de espera para receber intervenções motivacionais após a conclusão do grupo 1 .

4 semanas
Participantes na condição de EM (grupo 1) foram significativamente mais propensos a completar o tratamento intensivo (taxa de conclusão de 69\%) do que aqueles na condição de controle (grupo 2) (31\%). 


\section{continuaçãi da Tabela 1 - Artigos Revisados}

\author{
Estudo \\ Geller, J. et al. \\ (2011) \\ Katzman, M. A. et al. \\ (2010) \\ Wade, T. D. et al. \\ (2009) \\ Amostra \\ 113 participantes \\ diagnosticados com \\ transtornos da \\ alimen tação. \\ 225 participantes com \\ BN ou EDNOS. \\ 47 pacientes \\ internados em \\ unidade de \\ transtorno \\ alimentar com \\ diagnóstico de AN.
}

Grupo 1: 57

participantes submetidos a cinco sessões de RMT (uma sessão por semana) com duração de aproximadamente $60^{\prime}$ cada.

Grupo 2: 56 participantes submetidos ao tratamento usual e incluídos em uma lista de espera para receber a intervenção motivacional após a conclusão do grupo 1 .

Duração

Principais resultados
Grupo 1: MET individual (79 participantes) - 1 sessão semanal por 12 semanas.

Grupo 2: MET em grupo (73 pessoas) - 1 sessão semanal por 12 semanas.

Grupo 3: TCC em grupo ( 73 pessoas) - 1 sessão semanal por 12 semanas.
Grupo 1: 22

pacientes receberam 4 sessões de EM com duração de 60' cada + tratamento usual.

Grupo 2: 25

pacientes receberam somente 0 tratamento usual.

\author{
Observou-se que os \\ indivíduos que \\ receberam as cinco \\ sessões de RMT \\ (grupo 1) foram \\ menos propensos a \\ ter alta \\ ambivalência do \\ que aqueles da \\ condição de \\ controle (grupo \\ 2).
}

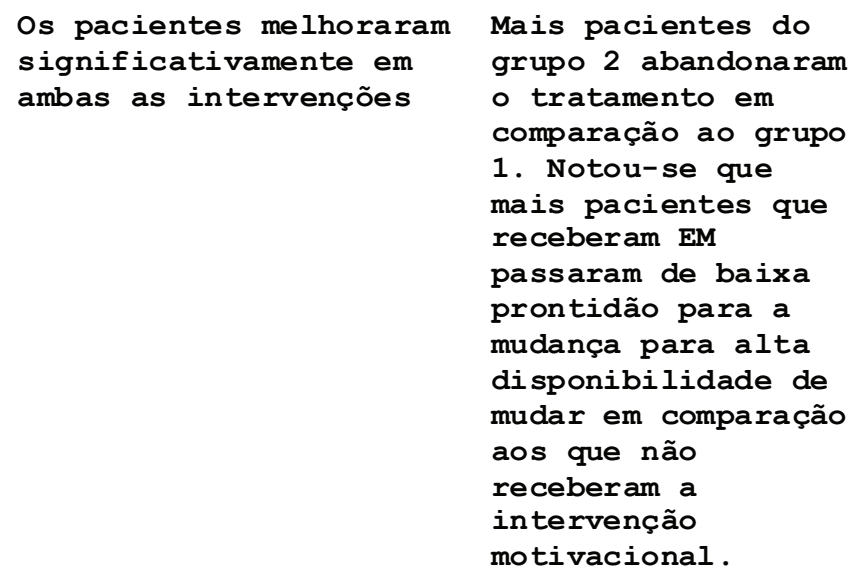




\section{continuaçãi da Tabela 1 - Artigos Revisados}

Es tudo

Amostra

$\begin{array}{ll}\text { Grupo } & \text { Grupo 1: uma } \\ \text { controle/ } & \text { sessão } \\ \text { experimental } & \text { individual de EM } \\ & \text { adaptada para } \\ & \text { transtornos da } \\ & \text { alimentação + } \\ & \text { manual de } \\ & \text { autoajuda. }\end{array}$

Duração

Principais resultados
108 mulheres diagnosticadas com TCAP.

Cassin, S.E. et al. (2008)

Dean, H. Y. et al. (2008)

42 pacientes diagnosticados com algum transtorno alimentar.

Grupo 1: 23 pacientes submetidos a 4 sessões de MET em grupo com duração aproximada de $1 \mathrm{~h} 25$ cada encontro.

Grupo 2: 19 pacientes submetidos Grupo 2: apenas manual de au toajuda. apenas ao tratamento usual.
Dunn, E. C. et al. (2006)

90 pacientes diagnosticados com BN Ou TCAP.

Grupo 1: 45 pacientes aleatoriamente submetidos a uma sessão de MET com duração de $1 \mathrm{~h}$ + manual de autoajuda.

Grupo 2: 45 pacientes que somente receberam - manual de autoajuda.

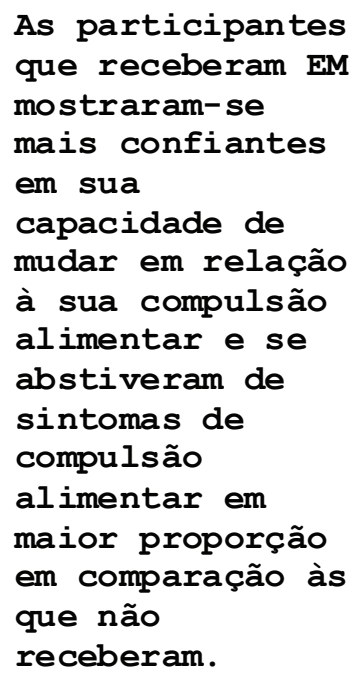

Os pacientes submetidos às sessões de MET (grupo 1) demonstraram maior motivação e engajamento para continuação do tratamento a longo prazo em comparação aos pacientes do grupo 2 .

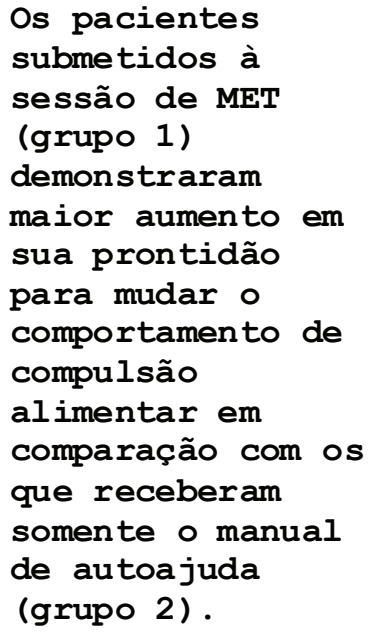


em grupo em comparação ao tratamento usual ou disponibilização de um manual de autoajuda no tratamento dos transtornos da alimentação, verificou-se que os indivíduos que receberam intervenções motivacionais foram significativamente mais propensos a aderir ao tratamento, menos propensos a manter alta ambivalência em relação à mudança e, consequentemente, mais motivados e engajados em modificar seus comportamentos "problema".

Os resultados da comparação entre os grupos experimental e controle de cada estudo aqui citado foram obtidos através do levantamento de dados obtidos por meio de instrumentos e questionários utilizados pelos pesquisadores com este fim. Tais instrumentos utilizados avaliam sintomas de transtornos alimentares, como o Eating Disorder Examination Questionnaire (EDE-Q) e a Short Evaluation of Eating Disorders (SEED); a prontidão para a mudança, como a URICA; motivação para mudar, como o Stages of Change Questionnaire for Eating Disorders (SOCQ-ED); e a autoeficácia, como o Weight Efficacy Lifestyle Questionnaire (WEL) e a Self Efficacy Scale (SES), dentre outros similares.

Ante o exposto, foi possível verificar que a EM e suas adaptações na MET e RMT demonstraram efetividade no tratamento dos transtornos da alimentação. Os resultados observados mostraram-se positivos por ter sido possível identificar uma maior motivação, engajamento, aumento da autoeficácia e da prontidão para mudança e consequente melhora na adesão ao tratamento em todos os grupos que foram submetidos a estas intervenções.

Para que o uso das intervenções motivacionais seja efetivo, o paciente deve ser encorajado a descobrir por si próprio as razões pelas quais ele deseja mudar. Ele deve ser encorajado a identificar suas dificuldades, seus motivos de preocupação, a sua disponibilidade para a mudança, e os fatores que ajudam ou prejudicam este processo. Para isso, é necessário que o terapeuta se utilize de perguntas abertas e do estilo de aconselhamento cliente-centrado (que é observado na EM e em suas adaptações), além de ele manter uma postura neutra, porém empática, evitando a confrontação - o que foi observado nos estudos analisados nesta revisão. Assim, possibilitou que fossem encontrados resultados que mostram efetividade no uso de intervenções motivacionais (MET, RMT e EM) no tratamento de pessoas com transtornos da alimentação. (Treasure \& Ward, 1997).

\section{Considerações finais}

Os objetivos desta revisão eram de examinar o contexto e a efetividade da utilização da EM e suas adaptações no tratamento de pessoas com transtornos da alimentação. Assim como de identificar possíveis limitações e/ou obstáculos neste processo. Em vista disto e com base nos resultados encontrados a partir da revisão de literatura realisada neste estudo, foi possível concluir que há efetividade na aplicação de tais intervenções motivacionais no tratamento dos transtornos da alimentação. Todos os estudos analisados demonstram significativo aumento na motivação, na prontidão para a mudança, na autoeficácia e, consequentemente, na adesão ao tratamento de pessoas diagnosticadas com algum transtorno da alimentação onde a EM e suas adaptações na MET e na RMT foram utilizadas.

O conhecimento proporcionado por esta revisão contribui para uma mais ampla compreensão a respeito do uso da Entrevista Motivacional e suas adaptações como uma importante e efetiva intervenção psicológica no tratamento dos transtornos da alimentação. Mesmo não tendo sido o objetivo deste estudo esgotar a discussão sobre este tema, é possível verificar que este trabalho oferece suporte teórico e empírico, por meio da análise dos estudos aqui abarcados, para identificar que a EM, a MET e a RMT são métodos clínicos possíveis e efetivos de serem utilizados no tratamento de transtornos para além da dependência química, ampliando o que inicialmente foi pensado no que diz respeito ao uso de tais intervenções motivacionais.

As limitações e obstáculos percebidos no desenvolvimento deste estudo se deram no sentido de identificar as características peculiares da receptividade e efetividade das intervenções motivacionais nos diferentes transtornos da alimentação, já que nenhum dos estudos procurou diferenciá-los em suas especificidades. Desta forma, sugere-se que, para futuros estudos, possa-se utilizar a EM de forma não associada a outras intervenções no tratamento de pessoas diagnosticadas com os diferentes transtornos da alimentação, observando sua efetividade em cada um destes transtornos isoladamente.

\section{REFERÊNCIAS BIBLIOGRÁFICAS}

\footnotetext{
Andretta, I., Meyer, E., Kuhn, R. P., Rigon, M. (2014). A entrevista motivacional no Brasil: uma revisão sistemática. Mudanças - Psicologia da Saúde, v.22, 2.ed, p.15-21. Disponível em: <https://www.metodista.br/revistas/ revistas-ims/index.php/MUD/article/viewFile/4622/4846>. Acesso em: 09 maio 2016.

Andretta, I.; Oliveira, M. S.; Zambom, L. F.; Dornelles, C. G. (2012). A entrevista motivacional: uma revisão de literatura atual. In: ANDRETTA, I.; OLIVEIRA, M. S. (Orgs). Manual prático de terapia cognitivo-comportamental. São Paulo: Casa do Psicólogo, p.521-536.

Blake, W., Turnbull, S., Treasure, J. (1997). Stages and processes of change in eating disorders. Implications for therapy. Clinical Psychology \&
} 


\section{REVISÃO SISTEMÁTICA DA APLICABILIDADE DA ENTREVISTA MOTIVACIONAL NO TRATAMENTO DOS TRANSTORNOS DA ALIMENTAÇÃO}

Psychotherapy, v.4, p.186-191, 1997. Disponível em: <http://onlinelibrary. wiley.com/doi/10.1002/(SICI) 1099-0879(199709)4:3\%3C186::AID-CPP128\%3E3.0.CO;2-5/epdf>. Acesso em: 07 maio 2016.

Cassin, S. E., Von Ronson, K., Heng, K., Brear, J., Wostowicz, A. (2008). Adapted Motivational Interviewing for women with Binge Eating Disorder: A Randomized Controlled Trial. Psychology of Addictive Behaviors, v.22, n.3, p.417-425, 2008. Disponível em: <https://psych.ucalgary.ca/manageprofile/ sites/psyc.ucalgary.ca.manageprofile/files/unitis/publications/220-40992/ adapted $\% 2$ BMI $\% 2$ Bfor $\% 2$ Bwomen $\% 2 B$ with $\% 2 B B E D$.pdf $>$. Acesso em: 08 maio 2016.

Dean, H. Y.; Touyz, S. W.; Rieger, E.; Thornton, C. E. (2008). Group motivational enhancement therapy as an adjunct to inpatient treatment for eating disorders: a preliminary study. European Eating Disorders Review, v.16, p.256-267. Disponível em: <http://onlinelibrary.wiley.com/doi/10.1002/ erv.851/abstract>. Acesso em: 04 maio 2016

Dunn, E. C.; Neighbors, C.; Larimer, M. (2006). Motivational Enhancement Therapy and self-help treatment for Binge Eaters. Psychology of Addictive Behaviors, v.20, n.1, p.44-52, Disponível em: < http://www.ncbi.nlm.nih. gov/pubmed/16536664>. Acesso em: 04 maio 2016.

Feld, R.; Woodside, B.; Kaplan, A.; Olmsed, M.; Carter, J. (2001). Pretreatment Motivational Enhancement Therapy for Eating Disorders: A Pilot Study. International Journal of Eating Disorders, v.29, p.393-400. Disponível em: $<$ http://onlinelibrary.wiley.com/doi/10.1002/eat.1035/abstract>. Acesso em: 04 maio 2016.

Finger, I. R.; Guedes, P. A. (2016). Diagnóstico e curso dos transtornos alimentares. In: FINGER, I. R.; OLIVEIRA, M. S. (Orgs). A prática da Terapia Cognitivo-Comportamental nos transtornos alimentares e obesidade. Novo Hamburgo: Synopsys Editora, p.24-36.

Geller, J., Brown, K. E., Srikameswaran, S. (2011). The efficacy of a brief motivational intervention for individuals with Eating Disorders: A Randomized Control Trial. International Journal of Eating Disorders, v.44, 6.ed., p.497-505. Disponível em: <http://www.ncbi.nlm.nih.gov/pubmed/20872758>. Acesso em: 04 maio 2016.

Hötzel, K., Von Brachel, R., Schmidt, U., Rieger, E. Kosfelder, J., Hechler, T., Schulte, D., Vocks, S. (2014). An internet-based program to enhance motivation to change in females with symptoms of an eating disorder: a randomized controlled Trial. Psychological Medicine, v.44, p.1947-1963, Disponível em: <http://www.ncbi.nlm.nih.gov/pubmed/24128818>. Acesso em: 04 maio 2016.

Katzman, M., Bara-Carril, N., Rabe-Hesketh, S., Troop, N., Treasure, J. (2010). A randomized controlled two-stage trial in the treatment of Bulimia Nervosa, comparing CBT Versus Motivational Enhancement in phase 1 followed by group Versus individual CBT in phase 2. Psychossomatic Medicine, v.72. Disponível em: <http://www.ncbi.nlm.nih.gov/pubmed/20668284>. Acesso em: 03 maio 2016.

Linde, K.; Willich, S. N. (2003). How objective are systematic reviews? Differences between reviews on complementary medicine. Journal of the Royal Society of Medicine, v.96, p.17-22. Disponível em: < http://www.ncbi.nlm. nih.gov/pubmed/12519797>. Acesso em: 09 maio 2016.

Miller, W. R.; Rollnick, S.(2001). Entrevista motivacional: preparando as pessoas para a mudança de comportamentos adictivos. Porto Alegre: Artmed Editora.

Miller, W. R.; Rollnick, S. (2009). Ten things that Motivational Interviewing is not. Behavioural and Cognitive Psychotherapy, v.37, p.129-140. Disponível em: < http://www.ncbi.nlm.nih.gov/pubmed/19364414>. Acesso em: 09 maio 2016.

Moher, D., Liberati, A., Tetzlaff, J., Altman, D. (2009). The PRISMA Group. Preferred Reporting Items for Systematic Reviews and Meta-Analyses: The PRISMA Statement. PLoS Med, v.6, t.ed., Disponível em: <http://www. prisma-statement.org >. Acesso em: 06 maio 2016.

Morgan, C. M., Vecchiatti, I. R., Negrao, A. B. (2002). Etiologia dos transtornos alimentares: aspectos biológicos, psicológicos e sócio-culturais. São Paulo: Revista Brasileira de Psiquiatria, v.24. Disponível em: <http://www. scielo.br/scielo.php?script=sci_arttext\&pid $=$ S1516-44462002000700005 $>$. Acesso em: 09 maio 2016.

Prochaska, J. O.; DiClemente, C, C. (1982). Transtheoretical therapy: toward a more integrative model of change. Psychotherapy: Theory, Research \& Practice, v.19, p.276-288. Disponível em: <http://www.hbftpartnership.com/ documents/uploadResources/TranstheoreticalT-Prochaska1982.pdf $>$. Acesso em: 09 maio 2016.

Prochaska, J. O., DiClemente, C., Norcross, J. C. (1992). In search of how people change: applications to addictive behaviors. American Psychologist, v.47, p.1102-1114. Disponível em: <http://www.ncbi.nlm.nih.gov/pubmed/1329589>. Acesso em: 09 maio 2016.

Sales, C. M. B.; Figlie, N. B. (2009). Revisão de literatura sobre a aplicação da entrevista motivacional breve em usuários nocivos e dependentes de álcool. Psicologia em Estudo, v.14, n.2. Disponível em: <http://www.scielo. br/scielo.php?script $=$ sci_arttext\&pid $=$ S1413-73722009000200014>. Acesso em: 09 maio 2016.

Szupzinski, K.; Oliveira, M. S. (2008). O modelo transteórico no tratamento da dependência química. Psicologia: Teoria e Prática, v.10, 1.ed, p.162-173. Disponível em: <http://pepsic.bvsalud.org/pdf/ptp/v10n1/v10n1a12. pdf>. Acesso em: 09 maio 2016.

Treasure, J.; Ward, A. (1997). A practical guide to the use of Motivational Interviewing in Anorexia Nervosa. London: European Eating Disorders Review, v.5, 2.ed., p.102-114. Disponível em: <http://onlinelibrary. wiley.com/doi/10.1002/(SICI)1099-0968(199706)5:2\%3C102::AID-ERV201\%3E3.0.CO;2-6/abstract>. Acesso em: 09 maio 2016.

Vella-Zarb, R. A.; Mills, J. S.; Westra, H. A.; Carter, J. C.; Keating, L. (2015). A randomized controlled trial of Motivational Interviewing 1 self-help Versus Psychoeducation 1 self-help for binge eating. International Journal of Eating Disorders, v.48, 3.ed., p.328-332. Disponível em: < http://onlinelibrary. wiley.com/doi/10.1002/eat.22242/abstract>. Acesso em: 09 maio 2016.

Wade, T. D.; Frayne, A.; Edwards, S.; Robertson, T.; Gilchrist, P. (2009). Motivational change in an inpatient anorexia nervosa population and implications for treatment. Australian and New Zeland Journal of Psychiatry, v.43, p.235243. Disponível em: < http://www.ncbi.nlm.nih.gov/pubmed/19221912>. Acesso em: 09 maio 2016.

Weiss, C. V.; Mills, J. S.; Westra, H. A.; Carter, J. C.(2013). A preliminary study of motivational interviewing as a prelude to intensive treatment for an eating disorder. Journal of Eating Disorders, v.34, 1.ed. Disponível em: <http://www.ncbi.nlm.nih.gov/pmc/articles/PMC4081789/>. Acesso em: 09 maio 2016.

Submetido em: 7-3-2017

Aceito em: 5-9-2017 